\title{
Identification of new rice cultivars and resistance loci against rice black-streaked dwarf virus disease through genome-wide association study
}

Zhiming Feng ${ }^{1,2+}$, Houxiang Kang ${ }^{3 \dagger}$, Mingyou Li ${ }^{1}$, Lihua Zou' ${ }^{1}$ Xiaoqiu Wang ${ }^{1}$, Jianhua Zhao ${ }^{1}$, Lang Wei ${ }^{1}$, Nana Zhou', Qianqian Li', Ying Lan ${ }^{5}$, Yafang Zhang ${ }^{1}$, Zongxiang Chen ${ }^{1}$, Wende Liư ${ }^{3}$, Xuebiao Pan ${ }^{1}$, Guo-Liang Wang ${ }^{3,4^{*}}$ (D) and Shimin Zuo ${ }^{1,2^{*}}$ (D)

\begin{abstract}
Background: The rice black-streaked dwarf virus (RBSDV) disease causes severe rice yield losses in Eastern China and other East Asian countries. Breeding resistant cultivars is the most economical and effective strategy to control the disease. However, few varieties and QTLs for RBSDV resistance have been identified to date.

Results: In this study, we conducted a genome-wide association study (GWAS) on RBSDV resistance using the rice diversity panel 1 (RDP1) cultivars that were genotyped by a 44,000 high-density single nucleotide polymorphism (SNP) markers array. We found that less than 15\% of these cultivars displayed resistance to RBSDV when tested under natural infection conditions at two locations with serious RBSDV occurrence. The aus, indica and tropical japonica sub-populations displayed higher RBSDV resistance than the aromatic and temperate japonica sub-populations. In particular, we identified four varieties that displayed stable levels of RBSDV resistance at all testing locations. GWAS identified 84 non-redundant SNP loci significantly associated with RBSDV resistance at two locations, leading to the identification of 13 QTLs for RBSDV resistance. Among them, aRBSDV-4.2 and qRBSDV-6.3 were detected at both locations, suggesting their resistance stability against environmental influence. Field disease evaluations showed that qRBSDV-6.3 significantly reduces RBSDV disease severity by $20 \%$. Furthermore, introgression of qRBSDV-6.3 into two susceptible rice cultivars by marker-assisted selection demonstrated the effectiveness of aRBSDV-6.3 in enhancing RBSDV resistance.
\end{abstract}

Conclusions: The new resistant cultivars and QTLS against RBSDV disease identified in this study provide important information and genetic materials for the cloning of RBSDV resistance genes as well as developing RBSDV resistant varieties through marker-assisted selection.

Keywords: Rice (Oryza sativa L.), Rice black-streaked dwarf virus (RBSDV) disease, Genome-wide association study (GWAS), Quantitative trait loci (QTLs), Resistance effect

\footnotetext{
* Correspondence: wang.620@osu.edu; smzuo@yzu.edu.cn

'Zhiming Feng and Houxiang Kang contributed equally to this work.

${ }^{3}$ State Key Laboratory for biology of plant diseases and insect pests/Institute

of plant protection, Chinese academy of Agricultural Sciences, Beijing

100093, China

'Jiangsu Key Laboratory of Crop Genetics and Physiology/ Key Laboratory of

Plant Functional Genomics of the Ministry of Education/ Jiangsu Key

Laboratory of Crop Genomics and Molecular Breeding, Agricultural College

of Yangzhou University, Yangzhou 225009, China

Full list of author information is available at the end of the article
} 


\section{Background}

The rice black-streaked dwarf virus (RBSDV) disease is spread by small brown planthoppers (Laodelphax striatellus Fallén, SBPH) (Shikata and Kitagawa 1977; Ruan et al. 1984). Typical symptoms of the RBSDV disease include severe dwarf shrinkage, dark-green and rigid leaves, and short white waxy or black-streaked stripes along the abaxial leaf surface and veins on the stem (Ruan et al. 1984; Zhou et al. 2008; Zhou 2013). Once the plants are infected, it is generally incurable; therefore, the RBSDV disease is also known as "cancer" in rice (Azuhata et al. 1993). In recent years, the RBSDV disease is expanding in Eastern China and other East Asian countries, resulting in severe yield losses (Sun et al. 2013; Zhou et al. 2015). Breeding resistant cultivars are believed to be the more economical and effective strategies to control the RBSDV disease compared to pesticides spraying to control the transmission vector SBPH (Zhou et al. 2015; Sun et al. 2017). However, only a few studies reported the mapping of resistance genes and quantitative trait loci (QTLs) to RBSDV to date (Pan et al. 2009; Wang et al. 2010; Zheng et al. 2012; Li et al. 2013; Zhou et al. 2015; Zhang et al. 2016; Sun et al. 2017).

The resistance of rice to RBSDV has been reported to be a quantitative trait controlled by QTLs or multiple genes (Pan et al. 2009; Wang et al. 2010; Zheng et al. 2012). Using recombinant inbred lines (RILs) derived from a cross between Koshihikari and Guichao2, Wang et al. (2010) detected a QTL for RBSDV resistance on chromosome 3 with resistant alleles from Koshihikari. $\mathrm{Li}$ et al. (2013) identified 3 QTLs for RBSDV resistance on chromosomes 6, 7, and 9 with resistant alleles from Minghui63 using a RIL population derived from a Zhenshan97/Minghui63 cross; the major QTL on chromosome 6 was further located at a $627.6-\mathrm{kb}$ interval. The resistance of the Tetep cultivar to RBSDV is controlled by 3 QTLs located on chromosomes 3, 10, and 11, respectively (Zhou et al. 2015). Recently, several more QTLs for RBSDV resistance were identified on chromosomes $1,6,8$, and 9 using a RIL population derived from a cross between IR36 and L5494 (Zhang et al. 2016). Sun et al. (2017) identified a highly resistant variety 9194 and mapped 4. QTLs for RBSDV resistance on chromosomes $3,6,9$, and 11 using an $F_{2: 3}$ population derived from a cross between 9194 and Suyunuo. In general, due to the lack of highly resistant resources and major QTLs for RBSDV resistance, the progress of genetic study and breeding of rice resistance to RBSDV is slow. Therefore, in order to breed resistant cultivars and reduce yield losses caused by RBSDV, it is urgent to identify highly resistant germplasm and to map major QTLs for RBSDV resistance using new mapping techniques.
With the advancement of sequencing technology, genome-wide association study (GWAS) using highdensity genome-wide single nucleotide polymorphisms (SNPs), has become a powerful and popular strategy for mining genes/QTLs controlling complex traits in plants (Buckler et al. 2009; Huang et al. 2010; Brachi et al. 2011; Zhao et al. 2011; Morris et al. 2013; Wang et al. 2016; Yano et al. 2016; Li et al. 2017). Compared to the traditional genetic linkage method that requires biparental mapping populations, GWAS is based on diverse natural populations and can detect multiple variants at an identified locus (Flint-Garcia et al. 2003). In rice, many genes/QTLs related to growth, development, biotic and abiotic stress tolerance have been detected by GWAS (Huang et al. 2010; Famoso et al. 2011; Zhao et al. 2011; Kang et al. 2016; Zhu et al. 2016; Li et al. 2017). The identification of allelic variations in phenotypic diversity germplasm collections will be of great practical significance to rice breeding. However, there is no report for the identification of QTLs associated with RBSDV resistance by GWAS.

The rice diversity panel 1 (RDP1), which consists of about 420 Oryza sativa accessions from 82 countries, is divided into six sub-populations (tropical japonica [TRJ], temperate japonica [TEJ], indica [IND], aus [AUS], aromatic $[\mathrm{ARO}])$ and admixture $[\mathrm{ADM}])$ by structure analysis, and contains a large number of phenotypic and genetic diversity (Zhao et al. 2011; Eizenga et al. 2014; Zhu et al. 2016). Importantly, the RDP1 was genotyped with about 44,000 high-quality SNPs, which provides the basis for GWAS (McCouch et al. 2010; Tung et al. 2010). Recently, the RDP1 has been used to identify many genes/QTLs related to diverse traits in rice, such as heading date, protein content, panicle number, seed number per panicle, blast resistance, sheath blight resistance, aluminum and ozone tolerance (Famoso et al. 2011; Zhao et al. 2011; Norton et al. 2014; Ueda et al. 2015; Kang et al. 2016; Chen et al. 2019).

In this study, the RBSDV resistance of the RDP1 cultivars were evaluated in the fields and then GWAS was used to identify the RBSDV QTLs in the rice genome. Several varieties with high RBSDV resistance levels and 13 QTLs for RBSDV resistance were identified. Our results will be useful for the identification of candidate genes controlling RBSDV resistance as well as developing RBSDV resistant rice varieties through markerassisted selection (MAS).

\section{Results}

Less than $15 \%$ of the RDP1 varieties showed partial resistance to RBSDV with disease incidence lower than $20 \%$

A total of 305 rice cultivars of the RDP1 and the two susceptible control cultivars were evaluated for RBSDV 
resistance after natural infection in Yutai County and Jinan City in Shandong Province (2013), Lian Yungang City (two testing locations, called lian-1 and lian-2) in Jiangsu Province (2013), and Kaifeng City in Henan Province (2014). The disease incidences (DIs) of the RBSDV disease and their frequency distributions for these cultivars are shown in Additional file 1: Table S1 and Fig. 1. The DIs of the susceptible control WLJ-1 were $20.5 \%, 19.2 \%$ and $22.6 \%$ at Jinan, Lian-1 and Lian2, respectively, while, those of WLJ-1 and HD-5 were $43.9 \%$ and $66.5 \%$ at Yutai and Kaifeng, respectively (Fig. 1a), indicating that the RBSDV disease at Yutai and Kaifeng was more prevalent than at Jinan, Lian-1 and Lian-2. We further compared the DIs of the susceptible controls at different positions in the fields in Yutai and Kaifeng, and found that the DIs were $39.5 \sim 50.5 \%$ for WLJ-1 and 62\% 72\% for HD-5 (Fig. $1 \mathrm{~b}$ and c), indicating that varieties at the different positions could receive similar doses of RBSDV. Furthermore, the DIs of the RDP1 varieties were mostly within $30 \%$ with average DIs between $10 \%-20 \%$ at Jinan, Lian-1 and Lian-2, while mostly between $20 \% \sim 70 \%$ and $40 \% \sim 100 \%$ with average DIs of $43.1 \%$ and $61.3 \%$ at Yutai and Kaifeng, respectively (Fig. $1 \mathrm{~d}$ and e). This result confirmed that the
RBSDV disease occurrence rates at Yutai and Kaifeng were higher than those at Jinan, Lian-1 and Lian-2. Taken together, we concluded that the DIs data from Yutai and Kaifeng were more suitable for further screening of resistant germplasm and identifying RBSDV resistance genes.

Based on the DIs data at the Yutai and Kaifeng locations, we found that most of the tested varieties were susceptible to RBSDV, that no completely immune varieties were detected, and that only $14.1 \%$ (Yutai) and 8.2\% (Kaifeng) of the tested varieties displayed partial resistance to RBSDV with a DI lower than 20\% (Fig. 1; Additional file 1: Table S1), suggesting that resistance to RBSDV of most varieties was minimal.

\section{Four varieties were identified with stable resistance to RBSDV}

From the DI data of all varieties evaluated at Yutai, Jinan, Lian-1 and Lian-2 in 2013, we identified 8 candidate resistant varieties with DI less than $10 \%$, including the previously reported resistant variety Minghui 63 (Fig. 2a; Li et al. 2013). These 8 varieties were further evaluated at Kaifeng in 2014; the DI of 4 of them (Byakkoku Y5006 SelN, Koshihikari, Kun-Min-Tsieh-Huran

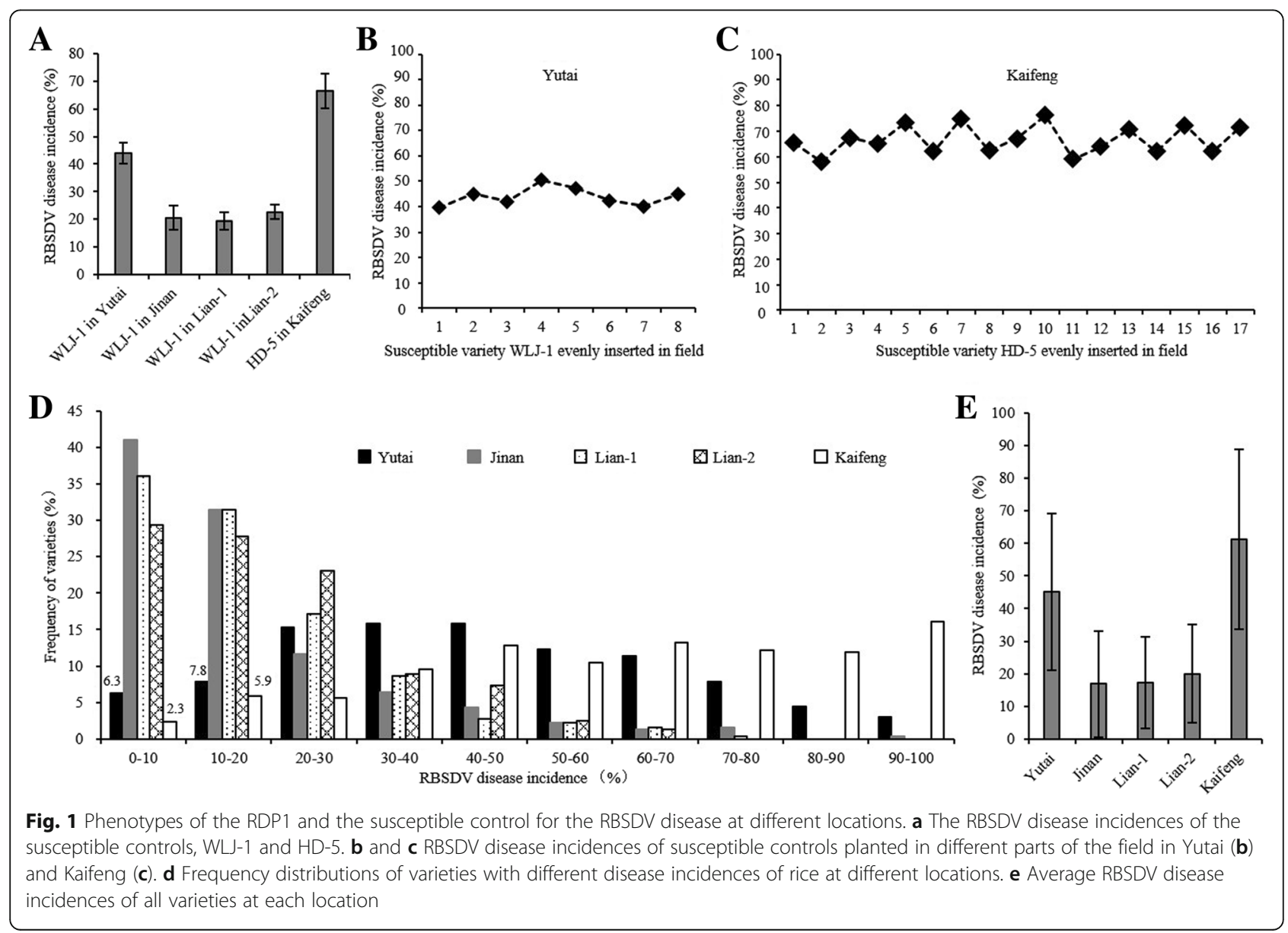




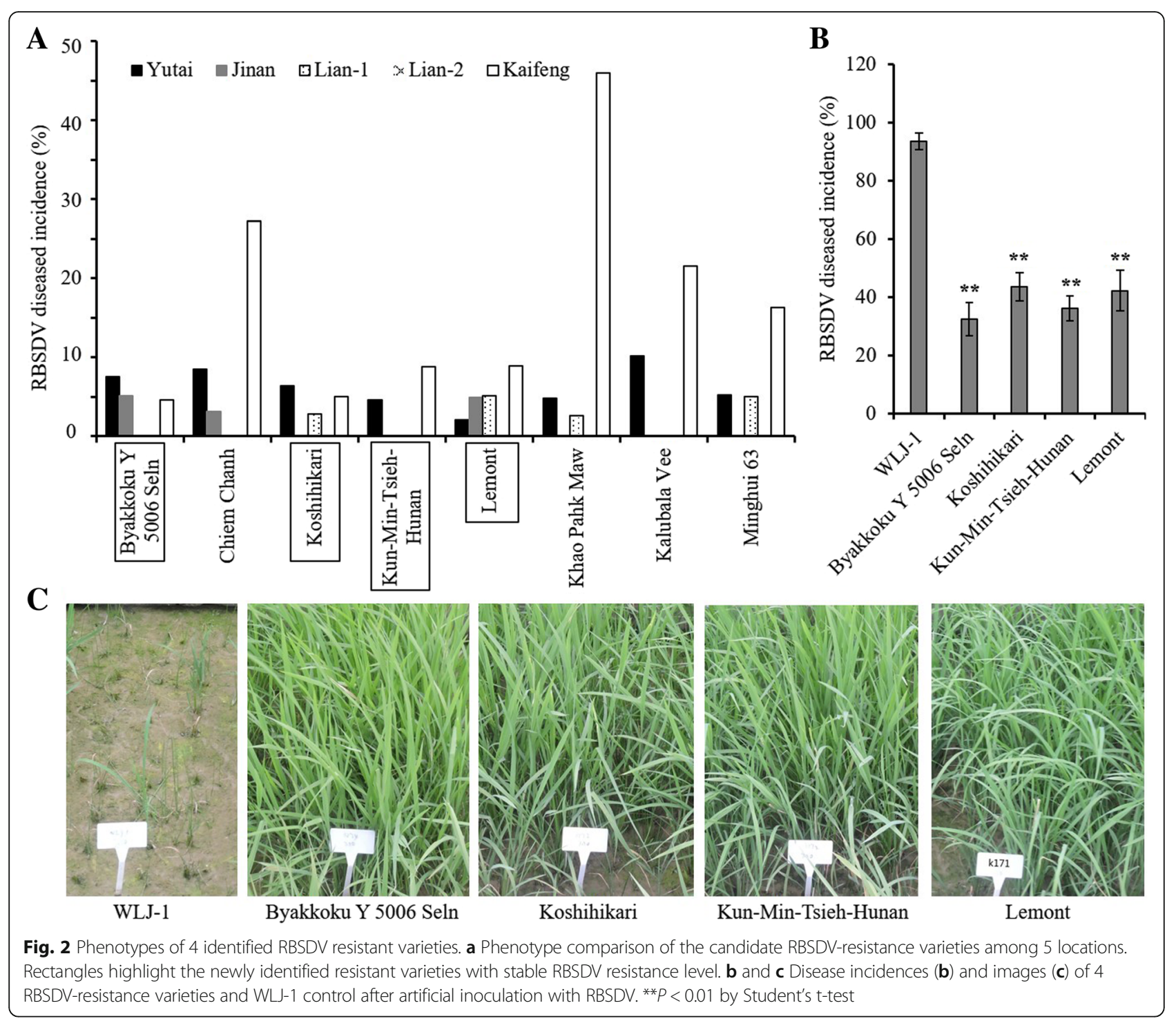

and Lemont) were lower than $10 \%$ while the remaining 4 (including Minghui 63) exceeded 10\% (Fig. 2a). Artificial inoculation was performed under controlled conditions to further validate the RBSDV resistance of the 4 resistant varieties. Compared to the susceptible control WLJ-1 yielded a DI of $93.6 \pm 2.9 \%$, these 4 varieties showed DI values of $32.4 \pm 5.6 \%, 43.5 \pm 4.9 \%, 36.0 \pm 4.3 \%$ and $42.2 \pm 6.9 \%$, respectively (Fig. $2 \mathrm{~b}$ and c), which are consistent with the field results. Collectively, we infer that the 4 varieties confer relatively high and stable resistance to RBSDV. Among them, we found Koshihikari and Lemont have been reported by the previous studies (Wang et al. 2010; Zheng et al. 2012), and the remaining two varieties, Byakkoku Y5006 SelN and Kun-MinTsieh-Huran, are newly identified in our study.

The Kaifeng location showed the most serious RBSDV occurrence as well as SBPH infestation in 2014 (Sun et al. 2017), presenting a good opportunity for us to determine whether the RBSDV resistance levels correlate with their resistance levels to SBPH, which transmits RBSDV. After preliminary observation by visual, we found that 4 of all plots/varieties apparently contained more $\mathrm{SBPH}$ than the other plots, and 7 plots/varieties contained less SBPH. For further quantifying the SBPH density, we counted the number of SBPH in these 11 varieties and other 17 varieties randomly selected (Table 1). We found that the 4 varieties did contain high density of SBPH with more than $20 \mathrm{SBPH}$ per plant, while the 7 varieties did harbor low density of SBPH with no more than $3 \mathrm{SBPH}$ per plant. The remaining 17 varieties, including the new RBSDV resistant variety Byakkoku Y5006 SelN, carried the number of SBPH range from 13 to 17; (Table 1). These suggest that most varieties showed similar response to $\mathrm{SBPH}$, while the 4 varieties were probably susceptible to $\mathrm{SBPH}$ and the other 7 had SBPH resistance although the resistant mechanism is unclear yet. 
Table 1 Disease incidences of the RBSDV disease and the number of SBPH landing on the varieties

\begin{tabular}{|c|c|c|c|c|c|}
\hline \multirow[t]{2}{*}{ Accession Name } & \multirow{2}{*}{$\begin{array}{l}\text { Country/region } \\
\text { of origin }\end{array}$} & \multirow[t]{2}{*}{ Sub-population } & \multirow{2}{*}{$\begin{array}{l}\text { Number of SBPH } \\
\text { (mean } \pm \text { SD) }\end{array}$} & \multicolumn{2}{|c|}{ RBSDV disease incidence at different locations } \\
\hline & & & & Kaifeng & Yutai \\
\hline Bulgare & France & TEJ & $25.3 \pm 5.8$ & $73.7 \%$ & $11.5 \%$ \\
\hline DZ 193 & Bangladesh & AUS & $20.8 \pm 2.4$ & $96.8 \%$ & $77.8 \%$ \\
\hline NPE 835 & Pakistan & TEJ & $22.8 \pm 4.2$ & $96.8 \%$ & $77.8 \%$ \\
\hline Ta Mao Tsao & China & TEJ & $23.6 \pm 3.6$ & $92.3 \%$ & $85.7 \%$ \\
\hline \multicolumn{4}{|c|}{ Average DI of varieties with high SBPH density } & $89.9 \% \pm 11.0 \%^{\mathrm{a}}$ & $63.2 \% \pm 34.7 \%$ \\
\hline Gerdeh & Iran & ADM & $16.6 \pm 3.1$ & $96.0 \%$ & $62.5 \%$ \\
\hline Jouiku 393G & Japan & TEJ & $15.1 \pm 2.3$ & $89.9 \%$ & $37.5 \%$ \\
\hline LAC 23 & Liberia & TRJ & $18.4 \pm 4.6$ & $51.9 \%$ & $44.4 \%$ \\
\hline Nova & United States & ADM & $13.9 \pm 1.5$ & $58.8 \%$ & $8.3 \%$ \\
\hline Romeo & Italy & TEJ & $15.2 \pm 3.3$ & $91.9 \%$ & $80.0 \%$ \\
\hline Byakkoku Y 5006 Seln & Australia & IND & $14.3 \pm 3.6$ & $4.5 \%$ & $7.5 \%$ \\
\hline Bergreis & Austria & TEJ & $16.7 \pm 5.2$ & $100.0 \%$ & - \\
\hline Azerbaidjanica & Azerbaijan & TEJ & $13.3 \pm 2.9$ & $100.0 \%$ & $100.0 \%$ \\
\hline Karabaschak & Bulgaria & TEJ & $15.8 \pm 4.4$ & $100.0 \%$ & $85.7 \%$ \\
\hline M. Blatec & Macedonia & ADM & $14.1 \pm 1.9$ & $97.5 \%$ & $100.0 \%$ \\
\hline Triomphe Du Maroc & Morocco & TEJ & $13.0 \pm 2.5$ & $82.1 \%$ & $60.0 \%$ \\
\hline Lusitano & Portugal & TEJ & $15.8 \pm 4.3$ & $96.3 \%$ & $25.0 \%$ \\
\hline WIR 3764 & Uzbekistan & TEJ & $13.7 \pm 3.3$ & $94.9 \%$ & $66.7 \%$ \\
\hline Okshitmayin & Myanmar & ADM & $14.3 \pm 4.1$ & $83.5 \%$ & $78.9 \%$ \\
\hline Sanbyang-Daeme & Korea & ADM & $15.2 \pm 2.7$ & $67.9 \%$ & $66.7 \%$ \\
\hline Heukgyeong & South Korea & TEJ & $17.4 \pm 4.6$ & $100.0 \%$ & $67.7 \%$ \\
\hline Bengal & United States & ADM & $16.8 \pm 3.9$ & $44.3 \%$ & $64.3 \%$ \\
\hline \multicolumn{4}{|c|}{ Average DI of varieties with moderate SBPH density } & $80.0 \% \pm 26.5 \%{ }^{b c}$ & $59.7 \% \pm 28.51 \%$ \\
\hline LD 24 & Sri Lanka & IND & $1.7 \pm 0.8$ & $30.0 \%$ & $7.4 \%$ \\
\hline Dee Geo Woo Gen & Taiwan & IND & $1.9 \pm 1.2$ & $81.5 \%$ & $69.8 \%$ \\
\hline Karkati 87 & Bangladesh & AUS & $2.1 \pm 0.8$ & $86.5 \%$ & $50.0 \%$ \\
\hline Chibica & Mozambique & TEJ & $2.8 \pm 1.5$ & $61.3 \%$ & $48.2 \%$ \\
\hline Kiang-Chou-Chiu & Taiwan & IND & $2.0 \pm 1.8$ & $63.8 \%$ & $21.8 \%$ \\
\hline Shangyu 394 & China & TEJ & $2.4 \pm 1.1$ & $68.4 \%$ & $62.5 \%$ \\
\hline Aijiaonante & China & IND & $1.5 \pm 1.3$ & $82.3 \%$ & $84.2 \%$ \\
\hline \multicolumn{4}{|c|}{ Average DI of varieties with low SBPH density } & $67.7 \% \pm 19.3 \%^{\mathrm{b}}$ & $49.1 \% \pm 26.9 \%$ \\
\hline
\end{tabular}

Different small letters indicate the difference on 5\% statistically significant difference. ADM, Admixture; AUS, aus; IND, indica; ARO, aromatic; TEJ, temperate japonica; TRJ, tropical japonica

We further compared the DIs of the varieties with high, moderate and low density of SBPH, and found that at Kaifeng location the varieties with low density of SBPH carried significantly stronger RBSDV resistance than those with high density of SBPH; while, no significant differences were found between varieties with high and moderate density of SBPH (Table 1). In addition, three varieties with low density of $\mathrm{SBPH}$ showed high DIs (by more than $80 \%$ ), and there were no significant differences on RBSDV DIs of the three types of varieties at Yutai location (Table 1). These implied that the SBPH density is not the critical factor on affecting variety resistance to RBSDV under the condition of serious SBPH and RBSDV occurrences in field. The 8 candidate RBSDV resistance varieties identified in 2013 contained no less than $6 \mathrm{SBPH}$ per plant (Additional file 2: Table S2), closing to that of the 17 varieties with moderate SBPH density. Except the Byakkoku Y5006 SelN, the 3 RBSDV resistant varieties, Koshihkari, Kun-Min-TsiehHuran and Lemont, confirmed in 2017 were found with $6.8 \pm 1.8,9.3 \pm 2.5$ and $8.4 \pm 1.3 \mathrm{SBPH}$, respectively. Taken together, considering the fact that the 4 RBSDV resistant varieties did not belong to the type of low $\mathrm{SBPH}$ density, we speculate that they most likely have 
ability to counter RBSDV but not SBPH, and would be useful for the identification of RBSDV resistance genes.

\section{Indica varieties carry the highest RBSDV resistance level among the six rice sub-populations}

To understand the differences in RBSDV resistance among sub-populations of the RDP1 cultivars, we analyzed the RBSDV resistance of the six rice subpopulations reported previously (Zhu et al. 2016). The analysis result showed that the AUS (average DI was $50.9 \%$ between Yutai and Kaifeng), IND (42.4\%) and TRJ (45.9\%) sub-populations were significantly more resistant than the ARO (72.8\%) and TEJ (66.8\%) sub-populations (Fig. 3). What's more, the IND sub-population carries the highest overall RBSDV resistance level among the six rice sub-populations.

Both novel and previously identified QTLs associated with RBSDV resistance were detected

To identify loci associated with RBSDV resistance, GWASs were carried out using the 44-K SNP data set and DI data of the RDP1 cultivars obtained from the Kaifeng and Yutai locations. Total of 35 and 54 SNP loci that were significantly associated with RBSDV resistance were found from the Kaifeng and Yutai test, repectively. The contribution of each significant association marker on phenotypic variance ranged from $5.03 \%$ to $9.94 \%$ (Fig. 4; Additional file 3: Table S3). These SNP loci were mainly distributed on chromosomes 1, 2, 3, 4, 6, 8 and 11 (Fig. 4). Among them, 9 and 6 associated regions that had at least 2 significant association markers from the Kaifeng and Yutai test were identified, respectively (Fig. 4). We designated these QTLs as qRBSDV-1.1,

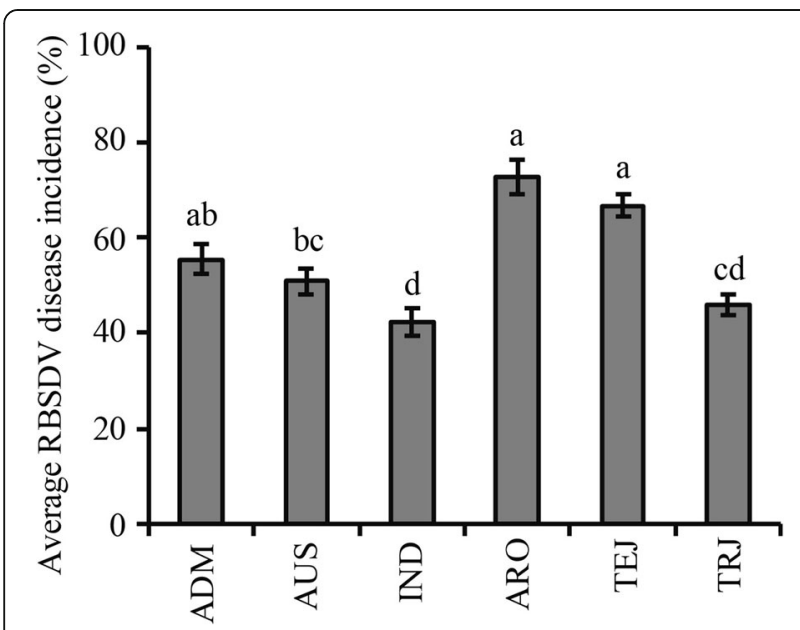

Fig. 3 Multi-comparison of RBSDV disease incidences of different sub-populations in the RDP1. Letters above columns indicate different statistical groups on $5 \%$ statistically significant difference. ADM, Admixture; AUS, aus; IND, indica; $A R O$, aromatic; TEJ, temperate japonica; TRJ, tropical japonica $q R B S D V-2.1, q R B S D V-3.1, q R B S D V-3.2, q R B S D V-3.3$, $q R B S D V-4.1, \quad q R B S D V-4.2, \quad q R B S D V-6.1, \quad q R B S D V-6.2$, $q R B S D V-6.3, q R B S D V-8.1, q R B S D V-8.2$, and $q R B S D V-$ 11.1, respectively (Fig. 4; Table 2). Most significantly, $q R B S D V-4.2$ and $q R B S D V-6.3$ were detected at both locations (Fig. 4), suggesting that these two QTLs were stable against the environmental influence. By comparing with the previously reported RBSDV QTLs, we found that $q R B S D V-3.2$ and $q R B S D V-3.3$ are collocated with $q R B S D V 3 b$ (Zheng et al. 2012), $q R B S D V-11.1$ collocated with $q R B S D V-11$ (Zhou et al. 2015), qRBSDV-6.3 partially overlapped with $q R B S D V 6$ detected by Sun et al. (2017), and the remaining 9 QTLs were not reported previously (Table 2 ).

qRBSDV-6.3 was detected with more association markers than others and is located in a region between SNPs id6010063 and id6010534 covering $1.9 \mathrm{Mb}$ (Additional file 3: Table S3; Table 2). In this region, five significant association markers (id6010277, id6010459, id6010472, id6010489 and id6010523) that covered a $439.154 \mathrm{~Kb}$ region were repeatedly detected at both test locations (Fig. 4; Additional file 3: Table S3), indicating that $q R B S D V-6.3$ is likely located within or near the interval of these 5 significant association markers. We identified 32 candidate genes in this $439.154 \mathrm{~Kb}$ region (Additional file 4: Table S4). Those candidate genes were classified into 17 category based on their annotation (Additional file 7: Figure S1). The four major categories were hypothetical protein (12), abscisic acid receptor PYL3-like (3), probable protein phosphatase $2 \mathrm{C}(2)$, and cyclic nucleotide-gated ion channel (2). For $q R B S D V-4.2$, although the most significant association markers (id4001630 and ud4000384, respectively) that detected were different at the two test locations, the distance between the two markers was about $1 \mathrm{MB}$ (Additional file 3: Table S3), implying that $q R B S D V-4.2$ is likely located within this $1 \mathrm{MB}$ region. In addition, $q R B S D V-1.1$ and $q R B S D V-6.2$ were also identified with higher degree of association (Fig. 4).

\section{qRBSDV-6.3 has obvious effects on reducing RBSDV disease severity}

To further assess the effect of $q R B S D V-6.3$ on RBSDV resistance, we employed the 5 stable significant association SNPs in the $q R B S D V-6.3$ region to distinguish varieties with and without the resistant alleles. The favorable/unfavorable alleles of these 5 SNPs (id6010277, id6010459, id6010472, id6010489 and id6010523) were $\mathrm{A} / \mathrm{C}, \mathrm{T} / \mathrm{A}, \mathrm{C} / \mathrm{T}, \mathrm{G} / \mathrm{A}$, and $\mathrm{T} / \mathrm{G}$, respectively. Their distributions are generally balanced in the RDP1 (Table 3). We found that the favorable allele of each SNP reduced the DI value by more than $15 \%$ at both test locations based on the relevant statistical parameters in the 


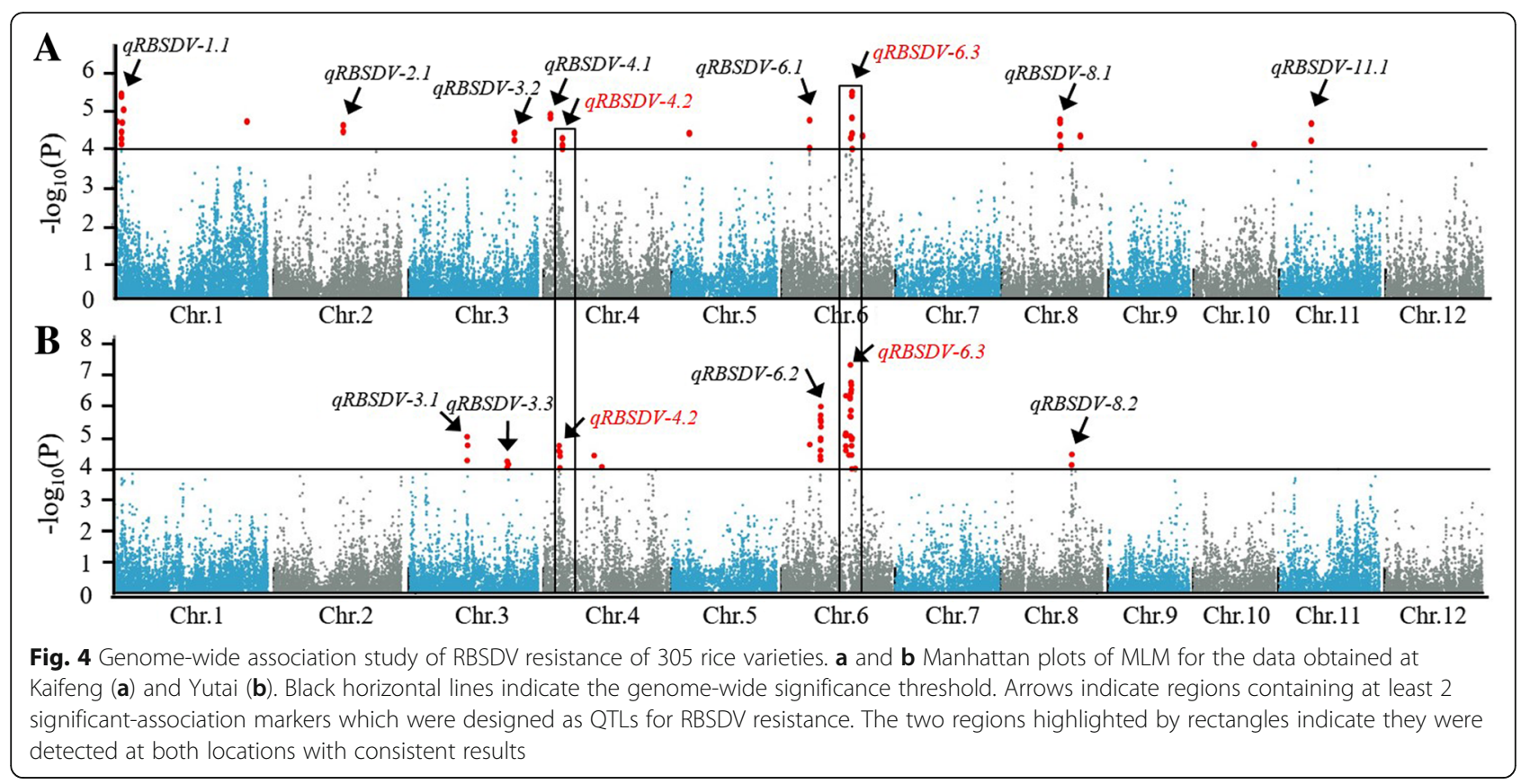

GWASs and the DI data between the favorable and unfavorable allele of each SNP (Table 3).

Further analysis showed that when the 5 SNP markers were combined there were 7 haplotypes in the RDP1 varieties, with the favorable haplotype being 'ATCGT' and the unfavorable being 'CATAG' (Additional file 5: Table S5). The number of varieties was 133 for the 'ATCGT' haplotype, 138 for 'CATAG', and 8 or less for each of the other 5 haplotypes (Additional file 5: Table S5). We found that there was no significant difference in the RBSDV resistance level between 'ATCGT' and 'CATAG' haplotypes (Additional file 5: Table S5). To test whether this might have resulted from population structure, we first examined what rice sub-populations each haplotype contained and then compared their RBSDV resistance levels. The unfavorable 'CATAG' haplotype contained AUS, IND, TEJ, TRJ and ADM sub-populations, while the favorable 'ATCGT' haplotype only contained ADM, TEJ and TRJ sub-populations but not AUS or IND sub-populations. These results implied that there exists a difference in the population structure of the RDP1 varieties between these two haplotypes. Thus, we were only able to examine among the ADM, TEJ and TRJ sub-populations. We found that among these three subpopulations the favorable 'ATCGT' varieties displayed significantly, or even extremely significantly, lower average DIs than the unfavorable 'CATAG' varieties under all five

Table 2 QTLs for the RBSDV disease

\begin{tabular}{|c|c|c|c|c|c|}
\hline QTL & Chromsome & Position & Top SNP marker & Loci reported $^{a}$ & Reference \\
\hline qRBSDV-1.1 & 1 & $2098423-2727493$ & id1001680 & & \\
\hline qRBSDV-2.1 & 2 & $19545113-29737544$ & id2007622 & & \\
\hline qRBSDV-3.1 & 3 & 16421475-16550808 & id3008188 & & \\
\hline qRBSDV-3.2 & 3 & $29724621-29737544$ & ud3001634 & aRBSDV $3 b$ & Zheng et al. 2012 \\
\hline qRBSDV-3.3 & 3 & 27699444-28028330 & id3012227 & aRBSDV $3 b$ & Zheng et al. 2012 \\
\hline qRBSDV-4.1 & 4 & 1875972-1876773 & id4000919 & & \\
\hline qRBSDV-4.2 & 4 & $4172738-5255769$ & id4001777 & & \\
\hline qRBSDV-6.1 & 6 & $7835229-7840016$ & id6004955 & & \\
\hline qRBSDV-6.2 & 6 & 10959905-11106570 & id6007011 & & \\
\hline qRBSDV-6.3 & 6 & 17993684-19865608 & id6010277 & qRBSDV6 & Sun et al. 2017 \\
\hline qRBSDV-8.1 & 8 & 16332728-16524733 & id8004300 & & \\
\hline qRBSDV-8.2 & 8 & 19756382-19826236 & id8005332 & & \\
\hline qRBSDV-11.1 & 11 & $9028442-9075159$ & id1 1003528 & qRBSDV-11 & Zhou et al. 2015 \\
\hline
\end{tabular}


Table 3 Statistic information of the five significant association SNP markers in Kaifeng and Yutai locations

\begin{tabular}{|c|c|c|c|c|c|c|c|c|c|c|c|}
\hline \multirow[t]{2}{*}{ Marker } & \multirow[t]{2}{*}{ Site } & \multirow[t]{2}{*}{ Allele } & \multicolumn{3}{|l|}{ Kaifeng } & \multicolumn{3}{|l|}{ Yutai } & \multicolumn{3}{|l|}{ Average } \\
\hline & & & Effect (\%) & Obs & Marker $\mathrm{R}^{2}$ & Effect (\%) & Obs & Marker $\mathrm{R}^{2}$ & Effect (\%) & Obs & Marker $\mathrm{R}^{2}$ \\
\hline \multirow[t]{2}{*}{ id6010277 } & 19426454 & $A$ & -16.63 & 142 & 5.44 & -19.66 & 137 & 9.94 & -16.93 & 142 & 8.73 \\
\hline & & C & 0.00 & 155 & & 0.00 & 152 & & 0.00 & 155 & \\
\hline \multirow[t]{2}{*}{ id6010459 } & 19737177 & $\mathrm{~T}$ & -17.82 & 151 & 6.04 & -15.17 & 146 & 5.66 & -15.36 & 151 & 6.96 \\
\hline & & A & 0.00 & 153 & & 0.00 & 150 & & 0.00 & 153 & \\
\hline \multirow[t]{2}{*}{ id6010472 } & 19739735 & C & -19.21 & 142 & 6.94 & -15.90 & 137 & 6.16 & -16.24 & 142 & 7.73 \\
\hline & & $T$ & 0.00 & 158 & & 0.00 & 155 & & 0.00 & 158 & \\
\hline \multirow[t]{2}{*}{ id6010489 } & 19785959 & G & -19.32 & 144 & 6.98 & -16.22 & 139 & 6.36 & -16.48 & 144 & 7.89 \\
\hline & & A & 0.00 & 160 & & 0.00 & 157 & & 0.00 & 160 & \\
\hline \multirow[t]{2}{*}{ id6010523 } & 19827029 & $\mathrm{~T}$ & -17.52 & 150 & 4.92 & -15.38 & 144 & 5.03 & -15.50 & 150 & 6.02 \\
\hline & & G & 0.00 & 154 & & 0.00 & 152 & & 0.00 & 154 & \\
\hline
\end{tabular}

Obs observation

locations, and the favorable 'ATCGT' haplotype reduced RBSDV disease severity by about 20\% under heavy RBSDV pressure at Kaifeng (Fig. 5). These results suggest that $q R B S D V-6.3$ had an obvious effect on RBSDV resistance.
Introgression of $q R B S D V-6.3$ into susceptible rice cultivars through MAS improves their RBSDV resistance

To validate the usability of $q R B S D V-6.3$, we introduced $q R B S D V-6.3$ from moderately resistant varieties Byakkoku Y 5006 Seln and Koshihikari into highly susceptible
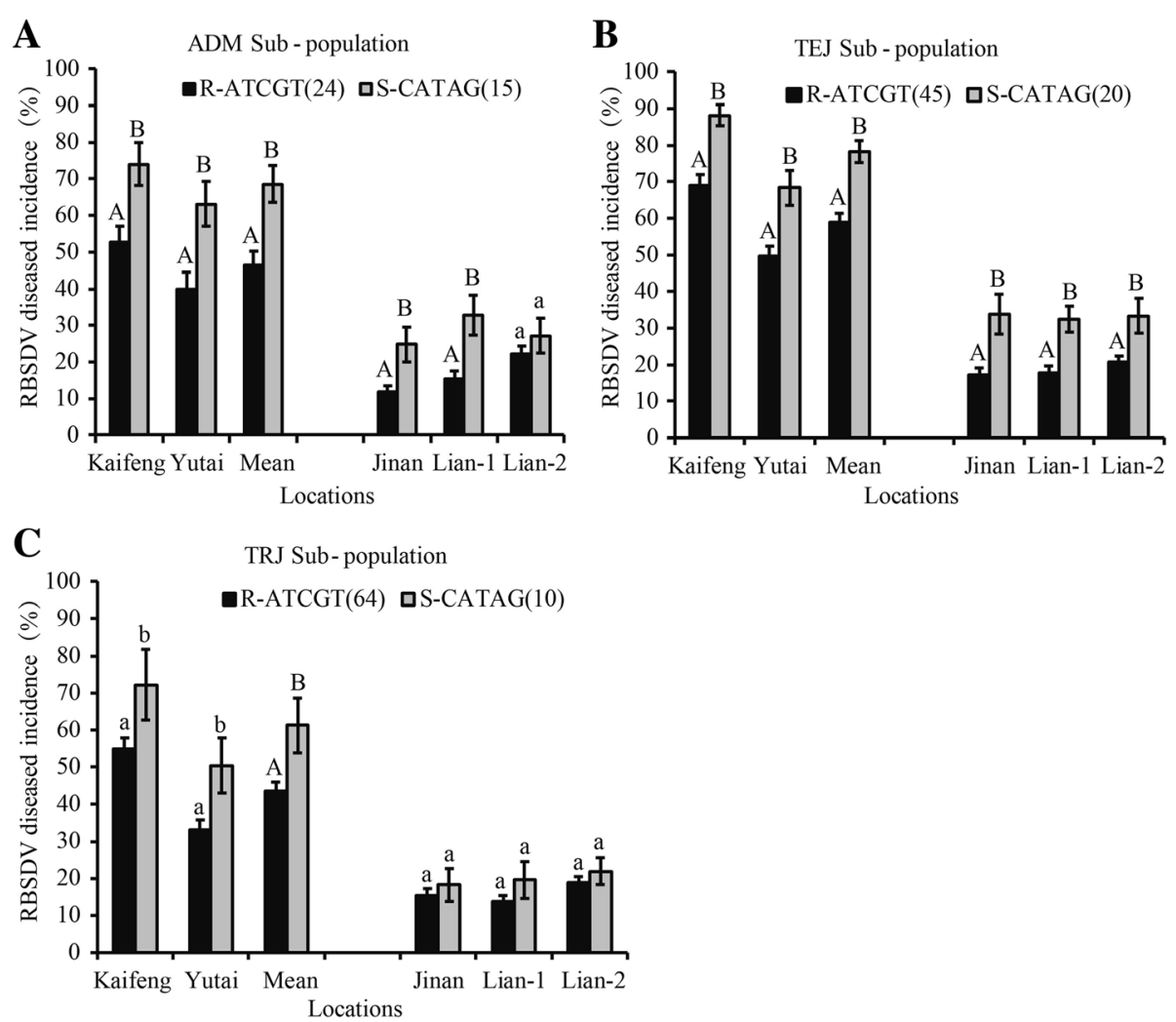

Fig. 5 Resistance comparison of the varieties with different haplotypes formed by 5 significant association SNP markers. a-c Resistance comparison of different haplotypes in ADM (a), TEJ (b) and TRJ (c) sub-populations. The haplotypes were formed from the 5 significant association SNP markers in the order of id6010277, id6010459, id6010472, id6010489 and id6010523 on chromosome 6. R-ATCGT and S-CATAG indicate resistant (favorable) and susceptible (unfavorable) haplotypes, respectively. The number following R-ATCGT or S-CATAG indicates the number of varieties having this haplotype. Different capital and lowercase letters above columns indicate 1\% and 5\% statistically significant difference, respectively. RBSDV, rice black-streaked dwarf virus; ADM, Admixture; TEJ, temperate japonica; TRJ, tropical japonica 
varieties WLJ-1 and HD-5. MAS was used to facilitate the development of three $\mathrm{BC}_{3} \mathrm{~F}_{2}$ populations: a Byakkoku Y 5006 Seln $x$ WLJ-1 $B_{3} \mathrm{~F}_{2}$ population, a Koshihikari $\mathrm{x}$ WLJ-1 $\mathrm{BC}_{3} \mathrm{~F}_{2}$ population, and a Koshihikari $\mathrm{x}$ HD-5 $\mathrm{BC}_{3} \mathrm{~F}_{2}$ population, using WLJ-1 or HD-5 as the recurrent parent. We obtained a total of 12 qRBSDV-6.3 introgressed homozygous lines and 7 homozygous lines without the $q R B S D V-6.3$ allele from the three $\mathrm{BC}_{3} \mathrm{~F}_{2}$ populations and evaluated them for RBSDV resistance. As shown in Fig. 6, the $q R B S D V-6.3$ positive lines clearly all displayed markedly higher resistance levels than the qRBSDV-6.3 negative lines in all three $\mathrm{BC}_{3} \mathrm{~F}_{2}$ populations, reducing their DIs roughly from 15 to $20 \%$ ( $q R B S D V-6.3$ negative) to $2-5 \%$ ( $q R B S D V-6.3$ positive). Taken together, these data further confirm that $q R B S D V-6.3$ is a reliable QTL against RBSDV and can be employed to improve rice RBSDV resistance.

\section{Discussion}

In recent years, the rice RBSDV disease has become such a serious rice disease that it has attracted great attention. Because RBSDV is not transmitted to plant offspring through the ovary, artificial infection by RBSDV using SBPH carrying RBSDV presents a great challenge, which leads to a serious lag in the research on identifying genetic resistance to RBSDV (Zhou 2013). So far, there are only a few reports about QTLs for RBSDV that all relied on natural infection in the field (Pan et al. 2009; Wang et al. 2010; Zheng et al. 2012; Li et al. 2013; Zhou et al. 2015; Zhang et al. 2016; Sun et al. 2017). In this study, we evaluated the RDP1 cultivars for resistance to RBSDV using the natural infection method at five testing locations where the RBSDV disease was prevalent in the previous years (Zhou 2013). Among these five testing locations, Kaifeng had the highest disease rate with the DI of the susceptible control HD-5 reaching $66.5 \%$, which is close to the DI of the artificial infection method (Zhou et al. 2015; Additional file 1: Table S1; Fig. 1). In addition, the DIs of the susceptible control WLJ-1 and HD-5 at different positions of the field in Yutai and Kaifeng were similar, showing that varieties at the different positions in the field received similar doses of RBSDV (Fig. 1). Therefore, the natural infection by RBSDV at Yutai and Kaifeng were successful, laying the foundation for our successful screening for resistant cultivars and for further genetic research of RBSDV resistance.

We identified 4 RBSDV-resistant cultivars (Byakkoku Y5006 SelN, Koshihikari, Kun-Min-Tsieh-Huran and Lemont) through screens based on natural infection (Fig. 2). To exclude those caused by insect resistance, we investigated the number of SBPH at the seedling stage, and found that the SBPH density did not significantly affect the RBSDV resistance phenotype (Table 1) and the 4 RBSDV-resistant varieties did not belong to the type of low SBPH density. So we speculate that the RBSDV resistances of them were most likely not dependent on resistance to SBPH and thus they most likely contain novel genes/alleles that confer resistance to RBSDV employing unexplored mechanisms. In the future study, we will evaluate the SBPH resistance level of them in the laboratory condition using the standard method including antibiosis test and non-preference test to verify our speculation.

To date, there are no reports dissecting the genetic architecture of resistance to RBSDV by using GWAS strategy. Here we identified 84 non-redundant SNP markers significantly associated with RBSDV resistance through GWAS with high density SNP markers, leading

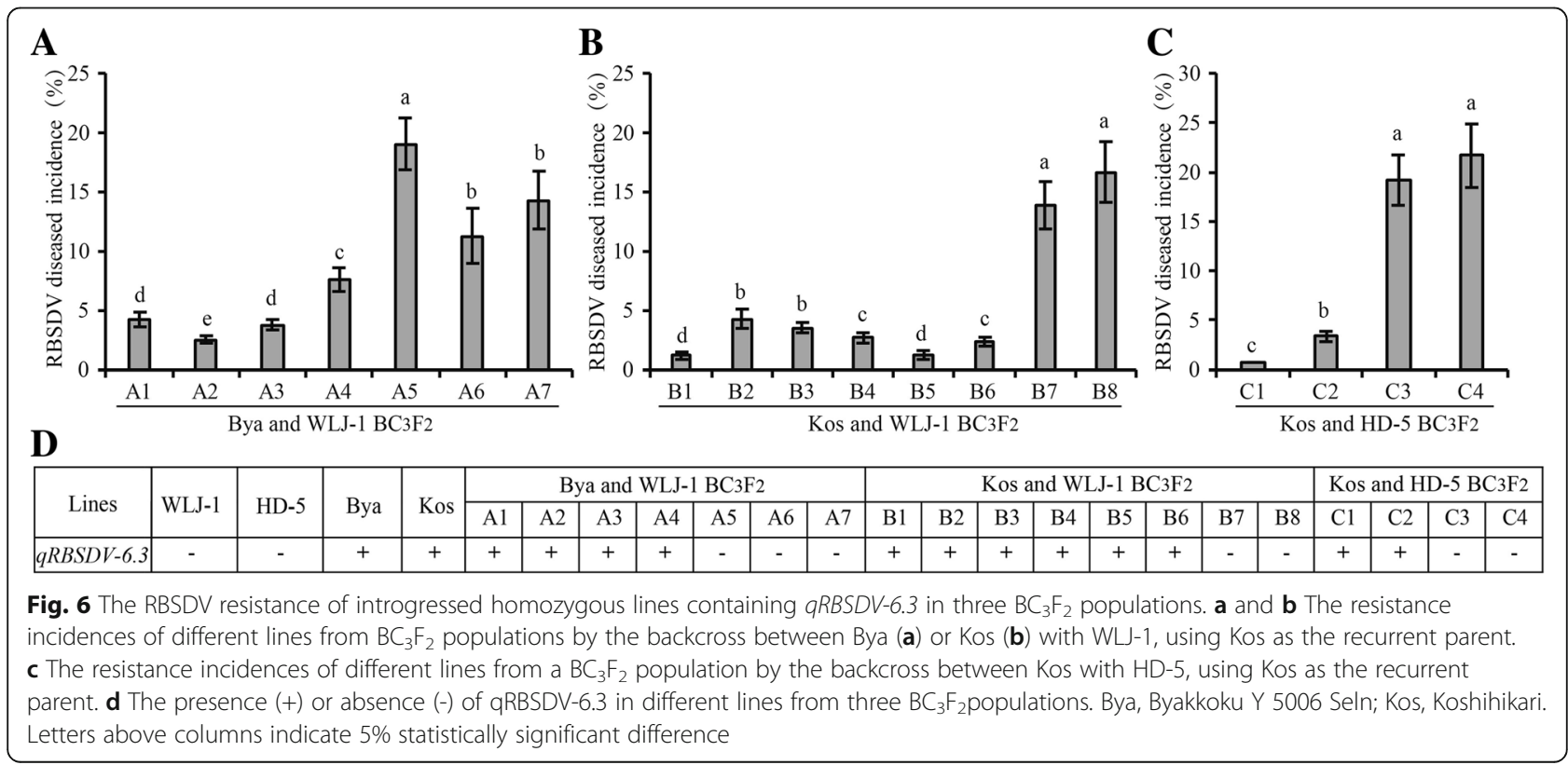


to the identification of 13 QTLs for RBSDV resistance (Fig. 4; Additional file 3: Table S3). Previously reported loci using traditional bi-parents QTL mapping strategy for RBSDV resistance were distributed on chromosomes 1, 3, 4, 6, 7, 8, 9, and 11 (Pan et al. 2009; Wang et al. 2010; Zheng et al. 2012; Li et al. 2013; Zhou et al. 2015; Zhang et al. 2016; Sun et al. 2017). By comparing the physical locations of them with 13 QTLs identified in our study, we found that qRBSDV-1.1, qRBSDV-2.1, $q R B S D V-2.1, q R B S D V-3.1, q R B S D V-4.1, q R B S D V-4.2$, $q R B S D V-6.1, q R B S D V-6.2, q R B S D V-8.1$, and $q R B S D V$ 8.2 did not locate to regions with known RBSDV resistance loci (Table 2), suggesting that these 9 QTLs are novel loci related to RBSDV resistance. Although $q R B S D V-3.2$ and $q R B S D V-3.3$ are collocated with $q R B S D V 3 b$ detected by Zheng et al. (2012), qRBSDV11.1 collocated with $q R B S D V-11$ detected by Zhou et al. (2015), and $q R B S D V-6.3$ partially overlapped with qRBSDV6 detected by Sun et al. (2017), these 4 QTLs are likely not controlled by the same genes or alleles as their corresponding reported RBSDV QTLs.

$q R B S D V-6.3$ was identified with the highest number of association SNP markers and degree of association among all regions (Fig. 4; Additional file 3: Table S3), and was estimated to reduce RBSDV disease severity by approximately $20 \%$ under severe RBSDV conditions (Fig. 5). Furthermore, when introgressed into susceptible rice cultivars through MAS, $q R B S D V-6.3$ significantly increased $\mathrm{RBSDV}$ resistance in all three $\mathrm{BC}_{3} \mathrm{~F}_{2}$ populations (Fig. 6). These results strongly indicate that $q R B S D V-6.3$ is a genuine major QTL and can be used in future rice breeding. Thus, $q R B S D V-6.3$ will be a focus for future research to mine resistance genes.

The greatest advantage of GWAS is that it can detect whether or not the tested cultivars carry favorable or unfavorable alleles at associated loci (Huang et al. 2010; Brachi et al. 2011; Zhao et al. 2011; Li et al. 2017), which is unachievable by previous linkage analysis based on double parents or a few parents. Thus, the results of GWAS can be more effectively combined with the practice of breeding. Using the information of the SNP marker loci associated with RBSDV resistance, we can select suitable parents according to needs, and achieve rapid transfer of the target loci by carrying out MAS (Li et al. 2018). Because RBSDV resistance is controlled by multiple genes, it is not practical to improve the overall RBSDV resistance level based only on marker-assisted stacking of multiple loci. It is still necessary to consciously increase the utilization frequency of resistant resources against RBSDV in traditional breeding. Our study showed that the AUS, IND and TRJ subpopulations are significantly more resistant than the ARO and TEJ sub-populations (Fig. 3). According to the RDP1 grouping by Zhao et al. (2011), most of the rice varieties grown in China belong to the IND and TEJ sub- populations. Therefore, in addition to using known resistance sources, we can appropriately increase the application frequency of cultivars in the AUS, IND and TRJ subpopulations in order to improve rice RBSDV resistance in China, especially for the TEJ sub-population rice varieties.

\section{Conclusions}

The new resistant cultivars and QTLs against RBSDV disease identified in this study provide important information and genetic materials for the cloning of RBSDV resistance genes as well as developing RBSDV resistant varieties through marker-assisted selection.

\section{Methods}

\section{Plant materials}

The three hundred and five $O$. sativa accessions screened for evaluation of RBSDV resistance and used in GWAS are part of the rice RDP1, and were provided by the Genetic Stocks-Oryza (GSOR) Collection, USDA ARS Dale Bumpers National Rice Research Center, USA. They represent six major sub-populations: TRJ (76 accessions), TEJ (77 accessions), IND (59 accessions), AUS (46 accessions), ARO (6 accessions) and ADM (42 accessions) (Zhao et al. 2011; Additional file 1: Table S1). Two rice japonica cultivars with low RBSDV resistance, Wulingjing1 (WLJ-1, high susceptible) and Huaidao 5 (HD-5, high susceptible), were selected as controls for evaluation of RBSDV resistance with the RDP1 (Li et al. 2013; Wang et al. 2014).

\section{Evaluation of RBSDV resistance by natural infection in the field and artificial inoculation}

The test locations were selected according to the occurrence of the RBSDV disease in the previous year. Experimental fields in Yutai County and Jinan City in Shandong Province (2013), Lian Yungang City (two testing locations, called lian-1 and lian-2) in Jiangsu Province (2013), and Kaifeng City in Henan Province (2014), respectively, were chosen for natural field evaluation in 2013 and 2014 due to prevalence of the RBSDV disease at these five locations in 2011 to 2013 (Zhou 2013). In 2013, the susceptible control cultivar WLJ-1 was added along each of the 40 tested varieties. In 2014, the susceptible control cultivar HD-5 was added along each of the 20 tested varieties. About 14 days before winter wheat harvest, 80 seeds per cultivar or line were sown in a $20 \times 10 \mathrm{~cm}$ plot in the experimental field encircled by wheat plants where SBPH lived. When the wheat were harvested, SBPH moved from the wheat plants into the neighboring rice seedlings. 7 days after sowing, all seedlings were thinned to about 60 plants per line. A month later, all seedlings were moved into an experimental field. Each line was replicated three times. During the experiment, the plants were cultured with normal field 
management and were not sprayed with antivirals or pesticides.

Seedling individuals with typical symptoms of the RBSDV disease were considered to be susceptible plants (Ruan et al. 1984), whereas those without typical symptoms were considered resistant plants. Resistance against RBSDV was evaluated based on the RBSDV disease incidence (DI) which was calculated as the number of RBSDV-infected plants divided by the total number of plants and multiplied by 100 . The survey of the incidence of the RBSDV disease was conducted at the peak tillering stage. The average DI was used for GWAS.

Artificial inoculation was performed according to the method described by Zhou et al. (2015). Non-virulent SBPH were fed on plants infected with RBSDV for 3 days to make them acquire the RBSDV. The SBPH were then transferred to rice seedlings in $5 \mathrm{~L}$ beakers and kept for 12 days to pass the circulative period of the virus. The random sampling and enzyme-linked immunoassay (ELISA) analysis were performed to estimate the proportion of viruliferous SBPH (Wang et al. 2006). The RBSDV viruliferous rate of SBPH was up to $32 \%$ (Additional file 8: Figure S2), indicating that there were enough virus sources. Sixty seeds per variety (three replicates) were sown into a $2 \mathrm{~L}$ beaker, then covered with tetoron gauze and fixed with vinyl tape. Fifty vigorous seedlings of each variety were kept for inoculation after eliminating weak seedlings at the 1.5-leaf stage. When the seedlings grow to the 2-leaf stage, 300 nymphs of RBSDV-carrying SBPH were released into each beaker. To make sure uniformity of the inoculation intensity, $\mathrm{SBPH}$ in each beaker were scattered three times daily for 3 days. The SBPH were subsequently removed from the dishes, and the seedlings were transplanted to a glasshouse at the Agricultural College of Yangzhou University. The RBSDV disease incidence was recorded as described in natural infection.

\section{GWAS analysis}

GWAS analysis was performed according to the methods previously described (Kang et al. 2016), based on the publicly available $44 \mathrm{~K}-\mathrm{SNP}$ data set of RDP1 accessions (Zhao et al. 2011). TASSEL 3.0 software and the mixed linear model (MLM) were used in GWAS (Bradbury et al. 2007). The MLM uses a joint kinship matrix and population structure model that can be described in Henderson's matrix notation (Henderson 1975). To control type 1 error, regions that had more than two SNPs with $P<1 \times 10^{-4}$ within a $200-\mathrm{kb}$ genomic window were considered for subsequent analysis. The Manhattan maps were plotted with PERL (Christiansen et al. 2012). $P<4.7 \times 10^{-5}(0.0001$ level $)$ was used as the significance threshold to determine significantly associated SNP markers. EMMAX was used to fit a standard linear mixed model (Kang et al. 2010). Manhattan and quantilequantile plots were produced by using the $R$ package (https://cran.rproject.org/web/packages/qqman/).

\section{Introgression of $q R B S D V-6.3$ through MAS}

To introgress $q R B S D V-6.3$ to WLJ-1/HD-5, we firstly crossed Byakkoku Y 5006 Seln / Koshihikari carrying qRBSDV-6.3 with WLJ-1/HD-5 and then the resultant hybrid $F_{1}$ s were backcrossed with parents WLJ-1/HD-5 three times. In each generation, two flanking markers (Additional file 6: Table S6) of $q R B S D V-6.3$ were deployed to select target recombinants. Finally, 4 homozygous $q R B S D V-6.3$-containing lines and 3 lines without $q R B S D V-6.3$ were obtained from the Byakkoku Y 5006 Sel and WLJ-1 $\mathrm{BC}_{3} \mathrm{~F}_{2}$ population, 6 homozygous $q R B S D V$-6.3-containing lines and 2 homozygous lines without $q R B S D V-6.3$ were obtained from the Koshihikari and WLJ-1 $\mathrm{BC}_{3} \mathrm{~F}_{2}$ population, and 2 homozygous $q R B S D V$-6.3-containing lines and 2 homozygous lines without $q R B S D V-6.3$ were obtained from the Koshihikari and HD-5 $\mathrm{BC}_{3} \mathrm{~F}_{2}$ population. The RBSDV resistance levels of these lines were evaluated in Kaifeng in 2018.

\section{Statistical analysis}

Microsoft Excel 2010 was used to manage the data. ANOVA and the Dunnett's multi-comparison test of the RBSDV disease scores among different varieties or subpopulations were carried out using the IBM SPSS version 16.0 (IBM Corp., Armonk, USA).

\section{Additional files}

Additional file 1: Table S1. Varieties used in assay for the RBSDV disease. (DOCX $64 \mathrm{~kb}$ )

Additional file 2: Table S2. Disease incidences of the RBSDV disease and the number of SBPH landing on the 8 candidate RBSDV resistance varieties identified in 2013. (DOCX $15 \mathrm{~kb}$ )

Additional file 3: Table S3. Information of SNP markers significantly associated with RBSDV disease resistance that were identified at Kaifeng and Yutai locations. (DOCX $31 \mathrm{~kb}$ )

Additional file 4: Table S4. Information on the 32 candidate SB resistant genes in the most possible location interval $(439.154 \mathrm{~kb})$ of qRBSDV-6.3. (DOCX $20 \mathrm{~kb}$ )

Additional file 5: Table S5. Phenotype of varieties with different haplotypes formed by the five association markers. (DOCX $19 \mathrm{~kb}$ )

Additional file 6: Table S6. Primers used in this study. (DOCX $18 \mathrm{~kb}$ ) Additional file 7: Figure S1. Classification of the 32 candidate genes in the most possible location interval (439.154 kb) of qRBSDV-6.3. (DOCX $161 \mathrm{~kb}$ )

Additional file 8: Figure S2. RBSDV detection by enzyme-linked immunoassay (ELISA). (DOCX 78 kb)

\section{Abbreviations}

ADM: Admixture; ARO: aromatic; AUS: aus; DI: Disease incidences; ELISA: Enzyme-linked immunoassay; GWAS: Genome-wide association study; HD-5: Huaidao 5; IND: indica; MAS: Marker-assisted selection; MLM: Mixed linear model; QTLs: Quantitative trait loci; RBSDV: Rice black-streaked dwarf virus; RDP1: Rice diversity panel 1; RILs: Recombinant inbred lines; 
SBPH: Small brown planthoppers; SNPs: Single nucleotide polymorphisms TEJ: temperate japonica; TRJ: tropical japonica; WLJ-1: Wulingjing1

\section{Acknowledgements}

We are thankful to Dr. Mawsheng Chern for critical comments on the manuscript.

\section{Authors' contributions}

FZM, KHX, PXB, WGL and ZSM conceived and designed the experiments. FZM, KHX, ZLH, LMY, WXQ, ZJH, WL, ZNN, LQQ, LY, ZYF, and LWD performed the experiments and analyzed the data. CZX was responsible for material plant and field management. FZM wrote the manuscript. WGL and ZSM revised the manuscript. All authors read and approved the manuscript.

\section{Funding}

This research was supported by the grants from the National Key Research and Development Program of China (2016YFD0100601), the National Key Technology Research and Development Program of the Ministry of Science and Technology of China (2015BAD01B01-5), Fok Ying Tung Education Fundation (151026), the Natural Science Foundation of China (31701057) and of Jiangsu province (BK20170487), China Postdoctoral Science Foundation (2017 M620227), the Innovation and Entrepreneurship Training Program for College Students of Jiangsu (201811117067Y) and a Project Funded by the Priority Academic Program Development of Jiangsu Higher Education Institutions (PAPD), respectively.

\section{Availability of data and materials}

All data supporting the conclusions of this article are provided within the article (and its Additional files)

\section{Ethics approval and consent to participate}

Not applicable.

\section{Consent for publication}

Not applicable.

\section{Competing interests}

The authors declare that they have no competing interests.

\section{Author details}

'Jiangsu Key Laboratory of Crop Genetics and Physiology/ Key Laboratory of Plant Functional Genomics of the Ministry of Education/ Jiangsu Key Laboratory of Crop Genomics and Molecular Breeding, Agricultural College of Yangzhou University, Yangzhou 225009, China. ${ }^{2}$ Jiangsu Co-Innovation Center for Modern Production Technology of Grain Crops, Yangzhou University, Yangzhou 225009, China. ${ }^{3}$ State Key Laboratory for biology of plant diseases and insect pests/Institute of plant protection, Chinese academy of Agricultural Sciences, Beijing 100093, China. ${ }^{4}$ Department of Plant Pathology, the Ohio State University, Columbus, OH 43210, USA. ${ }^{5}$ Institute of Plant Protection, Jiangsu Academy of Agricultural Sciences, Nanjing 210014, China.

\section{Received: 9 April 2019 Accepted: 1 July 2019}

Published online: 15 July 2019

\section{References}

Azuhata F, Uyeda I, Kimura I, Shikata E (1993) Close similarity between genome structures of rice black-streaked dwarf and maize rough dwarf viruses. J Gen Virol 74:1227

Brachi B, Morris GP, Borevitz JO (2011) Genome-wide association studies in plants: the missing heritability is in the field. Genome Biol 12:232

Bradbury PJ, Zhang Z, Kroon DE, Casstevens TM, Ramdoss Y, Buckler ES (2007) TASSEL: software for association mapping of complex traits in diverse samples. Bioinformatics 23:2633-2635

Buckler ES, Holland JB, Bradbury PJ, Acharya CB, Brown PJ, Browne C, Ersoz E, Flint-Garcia S, Garcia A, Glaubitz JC, Goodman MM, Harjes C, Guill K, Kroon DE, Larsson S, Lepak NK, Li H, Mitchell SE, Pressoir G, Peiffer JA, Rosas MO, Rocheford TR, Romay MC, Romero S, Salvo S, Sanchez VH, Da SH, Sun Q, Tian F, Upadyayula N, Ware D, Yates H, Yu J, Zhang Z, Kresovich S, McMullen MD (2009) The genetic architecture of maize flowering time. Science 325:714-718
Chen ZX, Feng ZM, Kang HX, Zhao JH, Chen TX, Li QQ, Gong HB, Zhang YF, Chen XJ, Pan XB, Liu WD, Wang GL, Zuo SM (2019) Identification of new resistance loci against sheath blight disease in rice through genome-wide association study. Rice Sci 26:21-31

Christiansen T, Foy BD, Wall L, Orwant J (2012) Programming Perl: unmatched power for text processing and scripting

Eizenga GC, Ali ML, Bryant RJ, Yeater KM, Mcclung AM, Mccouch SR (2014) Registration of the rice diversity panel 1 for genome wide association studies. J Plant Reg 8:109

Famoso AN, Zhao K, Clark RT, Tung CW, Wright MH, Bustamante C, Kochian LV, McCouch SR (2011) Genetic architecture of aluminum tolerance in rice (Oryza sativa) determined through genome-wide association analysis and QTL mapping. PLoS Genet 7:e1002221

Flint-Garcia SA, Thornsberry JM, Buckler ET (2003) Structure of linkage disequilibrium in plants. Annu Rev Plant Biol 54:357-374

Henderson CR (1975) Best Linear Unbiased Estimation and Prediction under a Selection Model. Biometrics 31 (2):423

Huang X, Wei X, Sang T, Zhao Q, Feng Q, Zhao Y, Li C, Zhu C, Lu T, Zhang Z, Li M, Fan D, Guo Y, Wang A, Wang L, Deng L, Li W, Lu Y, Weng Q, Liu K, Huang T, Zhou T, Jing Y, Li W, Lin Z, Buckler ES, Qian Q, Zhang QF, Li J, Han B (2010) Genome-wide association studies of 14 agronomic traits in rice landraces. Nat Genet 42:961-967

Kang KH, Sul JH, Service SK, Zaitlen NA, Kong S, Freimer NB, Sabatti C, Eskin E, (2010) Variance component model to account for sample structure in genome-wide association studies. Nature Genetics 42 (4):348-354

Kang $H$, Wang Y, Peng S, Zhang Y, Xiao Y, Wang D, Qu S, Li Z, Yan S, Wang Z, Liu W, Ning Y, Korniliev P, Leung H, Mezey J, McCouch SR, Wang GL (2016) Dissection of the genetic architecture of rice resistance to the blast fungus Magnaporthe oryzae. Mol Plant Pathol 17:959-972

Li A, Pan C, Wu L, Dai Z, Zuo S, Xiao N, Yu L, Li Y, Zhang X, Xue W (2013) Identification and fine mapping of aRBSDV-6MH, a major QTL for resistance to rice black-streaked dwarf virus disease. Mol Breeding 32:1-13

Li WT, Zhu ZW, Chern MS, Yin JJ, Yang C, Ran L, Cheng MP, He M, Wang K, Wang J, Zhou XJ, Zhu XB, Chen ZX, Wang JC, Zhao W, Ma BT, Qin P, Chen WL, Wang YP, Liu JL, Wang WM, Wu XJ, Li P, Wang JR, Zhu LH, Li SG, Chen XW (2017) A natural allele of a transcription factor in rice confers broad-spectrum blast resistance. Cell 170:114-126

Li Y, Xiao JH, Chen LL, Huang XH, Cheng ZK, Han B, Zhang QF, Wu CY (2018) Rice functional genomics research: past decade and future. Mol Plant 11: 359-380

Mccouch SR, Zhao KY, Wright M, Tung CW, Ebana K, Thomson M, Reynolds A, Wang D, Declerck G, Ali ML (2010) Development of genome-wide SNP assays for rice. Breeding Sci 60:524-535

Morris GP, Ramu P, Deshpande SP, Hash CT, Shah T, Upadhyaya HD, Riera-Lizarazu O, Brown PJ, Acharya CB, Mitchell SE, Harriman J, Glaubitz JC, Buckler ES, Kresovich S (2013) Population genomic and genome-wide association studies of agroclimatic traits in sorghum. Proc Natl Acad Sci U S A 110:453-458

Norton GJ, Alex D, Brett L, Elena Y, Lou GM, Pinson SRM, Lee T, Eizenga GC, Mcgrath SP, Zhao FJ (2014) Genome wide association mapping of grain arsenic, copper, molybdenum and zinc in rice (Oryza sativa L.) grown at four international field sites. PLoS One 9:e89685

Pan CH, Li AH, Chen ZX, Wu LB, Dai ZY, Zhang HX, Hu NS, Chen XJ, Zhang YF, Zuo SM, Pan XB (2009) Detection of QTL for resistance to rice black-streaked dwarf viral disease. Acta Agron Sin 35:2213-2217 (in Chinese with English abstract)

Ruan YL, Chen SX, Lin RF, Jiang WL, Jin DD (1984) Studies on rice black-streaked dwarf virus. J Zhejiang Agric Sci 4:185-187 (in Chinese with English abstract)

Shikata E, Kitagawa Y (1977) Rice black-streaked dwarf virus: its properties, morphology and intracellular localization. Virology 77:826-842

Sun F, Xu QF, Cheng ZB, Fan YJ, Zhou YJ (2013) Advances in rice black-streaked dwarf disease in China. Jiangsu J of Agr Sci 29:195-201 (in Chinese with English abstract)

Sun Z, Liu Y, Xiao S, Hu J, Pan G, He J, Xu T, Huang J, Qiu Z, Fan D, Zhang L, Liu $L$, Jiang $L$, Cheng $X$, Zhai H, Wan J (2017) Identification of quantitative trait loci for resistance to rice black-streaked dwarf virus disease and small brown planthopper in rice. Mol Breeding 37:72

Tung CW, Zhao K, Wright MH, Ali ML, Jung J, Kimball J, Tyagi W, Thomson MJ, Mcnally K, Leung H (2010) Development of a research platform for dissecting phenotype-genotype associations in Rice (Oryza spp.). Rice 3:205-217

Ueda Y, Frimpong F, Qi Y, Matthus E, Wu L, Höller S, Kraska T, Frei M (2015) Genetic dissection of ozone tolerance in rice (Oryza sativa L.) by a genomewide association study. J Exp Bot 66:293-306 
Wang BX, Jiang L, Chen LM, Lu BG, Wang Q, Le QT, Fan JW, Cheng XN, Zhai HQ, Xu DY, Wan JM (2010) Screening of rice resources against rice black-streaked dwarf virus and mapping of resistant QTL. Acta Agron Sin 36:1258-1264 in Chinese with English abstract

Wang BX, Song ZQ, Liu JB, Lu BG, Zhou ZL, Fan JW, Qin DR, Xu DY (2014) Screening and evaluation of rice from different provinces against rice blackstreaked dwarf virus disease. Southwest China J Agric Sci 27:2365-2369 (in Chinese with English abstract)

Wang X, Wang H, Liu S, Ferjani A, Li J, Yan J, Yang X, Qin F (2016) Genetic variation in ZmVPP1 contributes to drought tolerance in maize seedlings. Nat Genet 48:1233-1241

Wang ZH, Fang SG, Zhang ZY, Han CG, Li DW, Yu JL (2006) Development of an ID-ELISA for the detection of rice black-streaked dwarf virus in plants. J Virol Methods 134:61-65

Yano K, Yamamoto E, Aya K, Takeuchi H, Lo PC, Hu L, Yamasaki M, Yoshida S, Kitano H, Hirano K, Matsuoka M (2016) Genome-wide association study using whole-genome sequencing rapidly identifies new genes influencing agronomic traits in rice. Nat Genet 48:927-934

Zhang H, Ge Y, Wang M, Liu J, Si H, Zhang L, Liang G, Gu M, Tang S (2016) Mapping QTLs conferring resistance to rice black-streaked dwarf disease in rice ( Oryza sativa L). Euphytica 212:1-8

Zhao K, Tung CW, Eizenga GC, Wright MH, Ali ML, Price AH, Norton GJ, Islam MR, Reynolds A, Mezey J, McClung AM, Bustamante CD, McCouch SR (2011) Genome-wide association mapping reveals a rich genetic architecture of complex traits in Oryza sativa. Nat Commun 2:467

Zheng T, Yang J, Zhong W, Zhai H, Zhu L, Fan F, Ali AJ, Yang J, Wang J, Zhu J, Uzokwe VNE, Xu J, Li Z (2012) Novel loci for field resistance to black-streaked dwarf and stripe viruses identified in a set of reciprocal introgression lines of rice (Oryza sativa L.). Mol Breeding 29:925-938

Zhou GH, Wen JJ, Cai DJ, Li P, Xu DL, Zhang SG (2008) Southern rice blackstreaked dwarf virus: a new proposed Fijivirus species in the family Reoviridae. Chin Sci Bull 53:3677-3685

Zhou T (2013) The development of identification method and genetic study of resistance to rice black-streaked dwarf disease in rice varieties [PHD thesis]. Nanjing Agricultural University, Nanjing (in Chinese)

Zhou T, Du L, Wang L, Wang Y, Gao C, Lan Y, Sun F, Fan Y, Wang G, Zhou Y (2015) Genetic analysis and molecular mapping of QTLs for resistance to rice black-streaked dwarf disease in rice. Sci Rep 5:10509

Zhu D, Kang H, Li Z, Liu M, Zhu X, Wang Y, Wang D, Wang Z, Liu W, Wang GL (2016) A genome-wide association study of field resistance to Magnaporthe Oryzae in rice. Rice 9:44

\section{Publisher's Note}

Springer Nature remains neutral with regard to jurisdictional claims in published maps and institutional affiliations.

\section{Submit your manuscript to a SpringerOpen ${ }^{\circ}$ journal and benefit from:}

- Convenient online submission

- Rigorous peer review

- Open access: articles freely available online

- High visibility within the field

- Retaining the copyright to your article

Submit your next manuscript at $\boldsymbol{\nabla}$ springeropen.com 